\title{
Aggressive management of lung donors classified as unacceptable: Excellent recipient survival one year after transplantation
}

\author{
Michaela Straznicka, MD \\ David M. Follette, $\mathrm{MD}^{\mathrm{a}}$ \\ Mark D. Eisner, $\mathrm{MD}^{\mathrm{b}}$ \\ Peter F. Roberts, MD \\ Rebecca L. Menza, $\mathrm{RN}^{\mathrm{c}}$ \\ Wayne D. Babcock, $\mathrm{RN}^{\mathrm{c}}$
}

From the Department of Surgery, Division of Cardiothoracic Surgery, University of California, Davis, Medical Center, Sacramento, ${ }^{\mathrm{a}}$ and the Division of Pulmonary Medicine and Critical Care Medicine, University of California San Francisco ${ }^{\mathrm{b}}$ and the California Transplant Donor Network, ${ }^{\mathrm{c}}$ San Francisco, Calif.

Read at the Twenty-seventh Annual Meeting of The Western Thoracic Surgical Association, San Diego, Calif, June 20-23, 2001.

Received for publication June 28, 2001; revisions requested Aug 3, 2001; revisions received Nov 1, 2001; accepted for publication Dec 7, 2001

Address for reprints: David M. Follette, MD, Department of Surgery, University of California, Davis Medical Center, 2nd Floor, Sacramento, CA 95817-1418 (Email: david.follette@ucdmc.ucdavis.edu).

J Thorac Cardiovasc Surg 2002;124:250-8

Copyright (9) 2002 by The American Association for Thoracic Surgery

$0022-5223 / 2002 \$ 35.00+0 \quad \mathbf{1 2 / 6 / 1 2 3 8 1 3}$

doi: $10.1067 / \mathrm{mtc} .2002 .123813$
Objective: A dire shortage of lungs for transplantation exists. We hypothesized that aggressive organ procurement organization management of lungs usually rated as unacceptable (ratio of $\mathrm{PaO}_{2}$ to inspired oxygen fraction $<150$ ) might make them acceptable for transplantation. We also hypothesized that lungs from donors who died of trauma could be used for transplantation with recipient survival comparable with that seen with lungs from donors who died of nontraumatic causes.

Methods: From January, 1, 1995, through August 31, 2000, a total of 194 donors resulted in 228 lung transplants. Of these, 27 donors were deemed unacceptable for lung transplantation according to organ procurement organization protocol. We used the California Transplant Donor Network database to conduct a retrospective review of all 194 donors, including the 27 supposedly unacceptable donors who were treated with invasive monitoring (central venous pressure), methylprednisolone, fluid restriction, inotropic agents, bronchoscopy, and diuresis. We evaluated survivals at 30 days and 1 year of patients who received lungs rated as unacceptable and acceptable. In addition, we compiled data on recipient survival for a subgroup of 122 recipients with lungs from donors who died of trauma and compared these data with those of recipients who received lungs from donors who died of nontraumatic causes to see whether the donor's death by trauma resulted in higher recipient mortality.

Results: After aggressive organ procurement organization management, ratios of $\mathrm{PaO}_{2}$ to inspired oxygen fraction, central venous pressures, fluid balances, dopamine requirements, and chest radiographs of unacceptable donors according to organ procurement organization criteria were comparable with those of acceptable donors. There were no significant differences in recipient mortality between groups at 30 days or 1 year after transplantation. Moreover, no significant difference was found in mortalities of recipients who received lungs from donors who died of traumatic and nontraumatic causes.

Conclusion: Aggressive organ procurement organization management of donors initially considered unacceptable may increase the number of lungs available for transplantation.

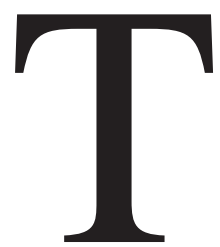

he incidence of end-stage lung disease has grown in the United States during the last 20 years, ${ }^{1}$ and lung transplantation is now an accepted treatment option for many of these patients. Last year, even though 877 lung transplants were performed in the United States, more than 2100 patients were added to the lung transplant waiting list. Almost 3500 patients are currently awaiting lung transplantation, and it is estimated that 600 of them will die before an organ becomes available. $^{2}$ A shortage of donor lungs remains the critical limiting factor. 
It is known that more than $80 \%$ of multiorgan donors do not have at least one lung used. Data released by the Association of Organ Procurement Organizations (AOPO) for the year 2000 reveal that among the nation's 25 largest organ procurement organizations (OPOs) the average lung procurement was only $13.4 \%$. The variability of percentages among OPOs was even more striking $(6.1 \%-27.1 \%) .^{3}$ Reasons for this wide discrepancy are multifaceted and include wide variations in donor management protocols, proximity of local lung transplantation centers, and OPO experience in maintaining lung donors. ${ }^{4,5}$

Traditionally, strict inclusion criteria for lung transplant donors have been required of OPOs by the transplantation centers. One approach to overcome this limitation has been liberalization of donor selection criteria to expand the available donor pool. ${ }^{6-8}$ Ideal lung donor selection criteria are age younger than 55 years; ABO compatibility, clear chest radiograph, $\mathrm{PaO}_{2}$ greater than $300 \mathrm{~mm} \mathrm{Hg}$ with an inspired oxygen fraction $\left(\mathrm{FIO}_{2}\right)$ of 1.0 and positive end-expiratory pressure of $5 \mathrm{~cm} \mathrm{H}_{2} \mathrm{O}\left(\mathrm{PaO}_{2} / \mathrm{FiO}_{2}\right.$ ratio $\left.>300\right)$, tobacco use history equivalent to less than 20 pack-years; absence of chest trauma, absence of aspiration or sepsis, lack of previous cardiopulmonary surgery, absence of organisms on sputum Gram stain, and absence of purulent secretions at bronchoscopy. ${ }^{9-11}$ Bhorade and colleagues ${ }^{6}$ studied donors who did not meet just one of these criteria, referring to them as "extended donors." They found that when these extended donors were used, the 1-year posttransplantation outcome was not adversely effected provided that oxygenation was maintained before lung procurement $\left(\mathrm{PaO}_{2} / \mathrm{FIO}_{2}\right.$ ratio maintained $>300$ ).

Other strategies to increase the number of donor lungs include attempts to improve the condition of donors considered unacceptable (those missing one or more of the ideal criteria and with $\mathrm{PaO}_{2} / \mathrm{FIO}_{2}$ ratios less than 150) by medical management. Traditionally in our OPO, lung donors with $\mathrm{PaO}_{2} / \mathrm{FIO}_{2}$ ratios less than 150 have been unquestioningly rejected, without consideration of the reversibility of the pulmonary insult. Several OPO centers have adopted aggressive management strategies to convert these initially unacceptable donors into acceptable ones.12,13 Little is known, however, about the long-term outcomes of the recipients of these previously unacceptable lungs that have been made acceptable by aggressive OPO management. One purpose of this review was to determine the outcomes when these donors were used.

How a donor dies may also play a role in recipient outcome. Traumatic wet lung was first described in 1945 and is recognized as a cause of respiratory dysfunction in patients with trauma. ${ }^{14}$ It has been hypothesized that trauma as a cause of death results in a greater number of early and late posttransplantation complications, although this hypothesis has never been carefully studied or validated.
In our retrospective study we evaluated the hypothesis that donor lungs with unacceptable $\mathrm{PaO}_{2} / \mathrm{FIO}_{2}$ ratios $(<150)$ can be made acceptable with aggressive OPO management and that 30-day and 1-year recipient outcomes with these lungs would not be significantly different than the outcomes of recipients with traditionally ideal lungs. We also compared the 30-day and 1-year outcomes of recipients with lungs from donors with traumatic and nontraumatic causes of death. We analyzed two groups of donors: acceptable donors, which included ideal donors and all donors who had one or more extended criteria but maintained $\mathrm{PaO}_{2} / \mathrm{FIO}_{2}$ ratios greater than 300 at the time of procurement, and unacceptable donors, those with a $\mathrm{PaO}_{2} / \mathrm{FIO}_{2}$ ratio less than 150 . We also compared the early and late results of recipients of lungs from donors with traumatic and nontraumatic causes of death.

\section{Methods}

From January 1, 1995, through August 31, 2000, a total of 194 donors resulted in 228 lung transplants. The lung donor group came from a potential pool of 1037 donors identified during the study. A retrospective analysis of the computerized database of the California Transplant Donor Network was used to review data for this study. Initial evaluation of all donors included age, smoking history, use of inhaled drugs, chest radiographic evaluation, measurement of $\mathrm{PaO}_{2} / \mathrm{FIO}_{2}$ ratio, and sputum Gram stains. Unacceptable donors were discriminated by initial poor oxygenation $\left(\mathrm{PaO}_{2} /\right.$ $\mathrm{FIO}_{2}<150$ ). Acceptable donors were those who met ideal criteria when OPO management began and maintained these criteria until procurement and those who had one or more criteria that classified them as expanded donors (Table 1) but at procurement had a normal chest radiograph and $\mathrm{PaO}_{2} / \mathrm{FIO}_{2}$ ratio greater than 300 . We compared the 30-day and 1-year survivals of recipients receiving lungs from unacceptable donors with those of recipients receiving lungs from acceptable donors. To determine whether death from trauma had an adverse effect on 30-day and 1-year lung recipient survivals, survivals of recipients of lungs from donors with traumatic causes of death were compared with those of recipients of lungs from donors with nontraumatic causes of death.

Aggressive management of all 27 unacceptable donors consisted of invasive central venous pressure (CVP) monitoring, methylprednisolone $(15 \mu \mathrm{g} / \mathrm{kg})$ immediately after declaration of brain death, fluid restriction, inotropes (dopamine drops) titrated for hemodynamic stability, bronchoscopy, diuresis, and radiographic review. The number of donors with a $\mathrm{PaO}_{2} / \mathrm{FIO}_{2}$ less than 150 who did not donate at least one lung was not available from our database. The decision to give steroids was based in part on transplantation center preferences. Type of transplantation performed (single-lung, double-lung, or heart-lung transplant) was recorded for all groups. Survival of recipients with lungs from the 27 unacceptable donors at 30 days and 365 days were compared with those of recipients of lungs from the 38 ideal donors and the entire group of 166 acceptable (expanded plus ideal) donors. Median survival was then calculated with $95 \%$ confidence intervals for each of the three groups. Information and transplantation follow-up were provided by the California Transplant Donor Network with a standard AOPO form. Data on hospital length of stay 
TABLE 1. Criteria for ideal and expanded lung donor groups

\begin{tabular}{|c|c|c|c|}
\hline & \multirow[b]{2}{*}{$\begin{array}{c}\text { Ideal criteria } \\
\text { ( } \mathbf{n}=\mathbf{3 8} \text { donors) }\end{array}$} & \multicolumn{2}{|c|}{ Extended criteria ( $\mathrm{n}=129$ donors) } \\
\hline & & Criterion & $\begin{array}{c}\text { Donors meeting } \\
\text { criterion (\%) }\end{array}$ \\
\hline $\mathrm{PaO}_{2} / \mathrm{FlO}_{2}$ ratio & $>300$ & $<300$ & 24.8 \\
\hline $\operatorname{Age}(y)$ & $<55$ & $>55$ & 7.8 \\
\hline Smoking (pack-year) & $<20$ & $>20$ & 2.3 \\
\hline Chest radiograph & Clear & Abnormal & 26.4 \\
\hline Bronchoscopy & Clean & $\begin{array}{l}\text { Presence of organisms } \\
\text { (cleared after } \\
\text { management) }\end{array}$ & 6.2 \\
\hline Inhaled drugs & No & Yes & 10 \\
\hline More than 1 extended criterion & Not applicable & Yes & 22.4 \\
\hline
\end{tabular}

*Ideal donors met all the ideal criteria during the entire management period. Donors in the expanded group met one or more of the extended criteria (as defined by Bhorade and colleagues ${ }^{6}$ ) during $\mathrm{OPO}$ management but maintained a $\mathrm{PaO}_{2} / \mathrm{FIO}_{2}$ ratio greater than 300 and had a normal chest radiograph at time of organ procurement.

\section{TABLE 2. Donor variables}

\begin{tabular}{lccc}
\hline & $\begin{array}{c}\text { Unacceptable } \\
\text { donor group } \\
\text { (n = 27) }\end{array}$ & $\begin{array}{c}\text { Acceptable } \\
\text { donor group } \\
\text { (n = 167) }\end{array}$ & $\begin{array}{c}\boldsymbol{P} \\
\text { value }\end{array}$ \\
\hline Age (y, mean \pm SEM) & $26.4 \pm 2$ & $31.2 \pm 1$ & .13 \\
$\begin{array}{l}\text { Gender (no.) } \\
\quad \text { Male }\end{array}$ & $21(77.8 \%)$ & $92(54.8 \%)$ & .034 \\
$\quad \begin{array}{l}\text { Female } \\
\text { Donor ethnicity (no.) }\end{array}$ & $6(22.2 \%)$ & $75(45.2 \%)$ & \\
$\quad$ White & $17(63 \%)$ & $109(65.1 \%)$ & .94 \\
$\quad$ Other & $10(37 \%)$ & $58(34.9 \%)$ & \\
Cause of death (no.) & $20(74.1 \%)$ & $79(47.6 \%)$ & .005 \\
$\quad \begin{array}{l}\text { Trauma } \\
\quad \text { Nontraumatic }\end{array}$ & $7(25.9 \%)$ & $88(52.4 \%)$ & \\
Steroid use (no.) & $27(100 \%)$ & $130(78 \%)$ & .0036 \\
\hline
\end{tabular}

after transplantation were also compared between groups. In addition, to see whether the recipient diagnosis affected survival differently according to the donor group, a Cox proportional hazard model was used. The two groups were compared with respect to selected variables - age, sex, cause of death, race, donor $\mathrm{ABO}$ type, and use of steroids immediately before procurement- to determine whether demographic, historical, or management factors were associated with increased likelihood of successful transplantation. Additional data for the unacceptable group included lowest $\mathrm{PaO}_{2} / \mathrm{FIO}_{2}$ ratio, final $\mathrm{PaO}_{2} / \mathrm{FIO}_{2}$ ratio, CVP when OPO management began, final CVP, fluid balance from donor arrival to intervention by OPO, fluid balance from OPO intervention to procurement, and dopamine dosages when OPO management began and at procurement.

Statistical analysis was performed with SPSS computer software (SPSS Inc, Chicago, Ill). Measurements are reported as mean \pm SEM. A 2-tailed Student $t$ test was applied to all numeric data. Categoric data were analyzed by $\chi^{2}$ analysis. Survival data were calculated by Kaplan-Meier analysis. A Cox proportional hazard model was used to discriminate variables within the study groups.
TABLE 3. Unacceptable versus ideal donor variables

\begin{tabular}{lccc}
\hline & $\begin{array}{c}\text { Unacceptable } \\
\text { donor group } \\
\text { (n= 27) }\end{array}$ & $\begin{array}{c}\text { Ideal } \\
\text { donor group } \\
\text { (n = 38) }\end{array}$ & $\begin{array}{c}\boldsymbol{P} \\
\text { value }\end{array}$ \\
\hline Age (y, mean \pm SEM) & $26.4 \pm 2$ & $28.7 \pm 1.9$ & .47 \\
$\begin{array}{l}\text { Gender (no.) } \\
\text { Male }\end{array}$ & $21(77.8 \%)$ & $22(57.9 \%)$ & .095 \\
$\quad$ Female & $6(22.2 \%)$ & $16(42.1 \%)$ & \\
Donor ethnicity (no.) & & & .93 \\
$\quad$ White & $17(63 \%)$ & $21(55.3 \%)$ & \\
$\quad$ Other & $10(37 \%)$ & $17(44.7 \%)$ & \\
Cause of death (no.) & $20(74.1 \%)$ & $2(55.3 \%)$ & .121 \\
$\quad \begin{array}{l}\text { Traumatic } \\
\quad \text { Nontraumatic }\end{array}$ & $7(25.9 \%)$ & $17(44.7 \%)$ & \\
Steroid use (no.) & $27(100 \%)$ & $27(71.1 \%)$ & .002 \\
\hline & & & \\
\hline
\end{tabular}

\section{Results}

Comparisons of unacceptable, ideal, and acceptable donors revealed that the unacceptable donors were predominantly male $(P=.034)$ and the cause of death was predominantly trauma $(P=.005$; Table 2$)$. There were no statistically significant differences in ethnicity, age, or ABO blood type among unacceptable and acceptable groups. All unacceptable donors received methylprednisolone after declaration of brain death, whereas only $78 \%$ of the acceptable donors received steroids ( $P=.0036$; Table 2$)$. The ideal donors (Table 3) did not differ from the unacceptable donors with respect to gender or ethnicity, but significantly fewer of the ideal donors than the unacceptable donors received steroids $(P=.002)$. Dopamine was titrated to minimal doses to maintain organ perfusion (Table 4), and dopamine requirements had decreased significantly by the time of procurement $(P<.005)$. In the unacceptable donor group, $\mathrm{PaO}_{2} /$ $\mathrm{FIO}_{2}$ ratios before OPO management were low; after management, they rose significantly $(P=.005)$. Initial CVP measurements were high before OPO intervention; from the 
TABLE 4. Results of management of the unacceptable donor group

\begin{tabular}{|c|c|c|c|}
\hline Variable ( $n=27$ donors) & $\begin{array}{c}\text { Before OPO } \\
\text { management }\end{array}$ & $\begin{array}{c}\text { At organ } \\
\text { procurement }\end{array}$ & $\begin{array}{c}P \\
\text { value }\end{array}$ \\
\hline $\begin{array}{l}\mathrm{PaO}_{2} / \mathrm{FiO}_{2}(\text { mean } \pm \mathrm{SEM})^{*} \\
\mathrm{FlO}_{2}(\text { mean } \pm \mathrm{SEM})^{*}\end{array}$ & $\begin{array}{c}103 \pm 6 \\
86 \% \pm 5 \%\end{array}$ & $\begin{array}{c}463 \pm 11 \\
100 \%\end{array}$ & $<.001$ \\
\hline CVP $(\mathrm{mm} \mathrm{Hg}$, mean \pm SEM) & $11.3 \pm 0.9$ & $6.7 \pm 0.4$ & $<.05$ \\
\hline $\begin{array}{l}\text { Net fluid balance (L, mean } \pm \\
\text { SEM) }\end{array}$ & $4.1 \pm 1.3$ & $-1.7 \pm 0.8$ & .008 \\
\hline $\begin{array}{l}\text { Dopamine requirement }(\mu \mathrm{g} / \\
[\mathrm{kg} \cdot \min ], \text { mean } \pm \mathrm{SEM})\end{array}$ & $15 \pm 0.1$ & $5.2 \pm 0.6$ & .001 \\
\hline $\begin{array}{l}\text { Abnormal chest radiograph } \\
\text { (no.) }\end{array}$ & $20 / 26$ & $0 / 26$ & .05 \\
\hline
\end{tabular}

*Positive end-expiratory pressure of $5 \mathrm{~mm} \mathrm{Hg}$.

time of OPO management to the time of organ procurement, CVP measurements dropped significantly $(P<.05)$. Fluid balance before OPO management was positive $(>4 \mathrm{~L})$; after management, it became negative $(P<.008)$. Chest radiographs were interpreted by an attending radiologist as having abnormal findings initially for most unacceptable donors; all radiographs showed subsequent correction to a normal appearance at the time of procurement $(P<.051)$. There were also no differences in 30-day and 1-year survivals between donors who received low doses $(<20 \mu \mathrm{g} /$ $[\mathrm{kg} \cdot \mathrm{min}])$ or high doses $(>20 \mu \mathrm{g} /[\mathrm{kg} \cdot \mathrm{min}])$ of dopamine during management (Table 5). Hospital stay averaged $16.06 \pm 1.4$ days in the unacceptable donor group. This compared favorably with $16.5 \pm 1.9$ in the ideal group and $20 \pm 1.9$ in the extended group. The data for hospital length of stay were not statistically significantly different among groups.

Recipient survivals at 30 days and 1 year were not different among the donor groups (Tables 5, 6, and 7 and Figure 1). The median survivals for all recipients were not different among groups ( $P=.70$ by log-rank test; Figure 1$)$. We believe that the reason for the slight drop in the ideal donor group survival at 1 year $(79.1 \%)$ was that all heartlung transplants came from this group (Table 6). It is important to note that all donors from all groups went to the operating room with a normal chest radiograph and a $\mathrm{PaO}_{2} /$ $\mathrm{FIO}_{2}$ ratio greater than 300 . The numbers of extended criteria that were met by each of our expanded donors at the time of OPO management are listed in Table 1. Twenty-two percent of these donors had more than one extended criterion, with $\mathrm{PaO}_{2} / \mathrm{FIO}_{2}$ ratio and an abnormal radiograph being the most common combination (13.9\%).

The recipients of organs from donors with traumatic causes of death had the same 30-day and 1-year survivals as did the recipients of lungs from donors who died of nontraumatic causes (Table 7; Figure 2). There were statistically more male donors with trauma as the cause of death, and the average age was lower among the donors with
TABLE 5. Recipient survivals at 30 days and 1 year related to dopamine dosage in unacceptable donor group

\begin{tabular}{|c|c|c|c|}
\hline Dopamine dose & $\begin{array}{c}\text { High dose } \\
(>20 \mu \mathrm{g} /[\mathrm{kg} \cdot \min ])\end{array}$ & $\begin{array}{c}\text { Low dose } \\
(<20 \mu \mathrm{g} /[\mathrm{kg} \cdot \mathrm{min}])\end{array}$ & $\begin{array}{c}P \\
\text { value }\end{array}$ \\
\hline Survival at $30 \mathrm{~d}$ & $83.3 \%$ & $95.5 \%$ & .279 \\
\hline Survival at 1 y & $83.3 \%$ & $90.9 \%$ & .602 \\
\hline
\end{tabular}

trauma as the cause of death than among the ideal and acceptable donor groups.

Recipient diagnosis (Tables 8 and 9) did not affect survival according to the donor lung group. The 95\% confidence interval for the Cox proportional hazard model indicated no statistical relationship between donor group and recipient survival controlling for recipient diagnosis.

\section{Discussion}

Aggressive OPO management of unacceptable donors resulted in improved oxygenation, hemodynamics, and chest radiographs at the time of lung procurement. The resultant organs were successfully transplanted without compromise of either 30-day or 1-year graft survival relative to lungs from both the ideal group and the acceptable group of donors. Expanded group donors had one or more criteria that would not have been traditionally approved; our acceptable group included ideal and expanded donors. At the time of procurement all donors had normal chest radiographs and a $\mathrm{PaO}_{2} / \mathrm{FIO}_{2}$ ratio greater than 300 . The ability to reverse the effects of aggressive fluid resuscitation, early pulmonary edema, and inflammation allowed these organs to be transplanted without adverse short- or long-term effects on the recipients.

The limitations of our study include the retrospective design, the small sample size, and the fact that all transplants were not performed at the same institution. The data were collected by a single OPO that serves a large geographic area with a number of transplantation centers. At the time of this study, standardized protocols had not been implemented between transplantation centers with respect to preoperative, intraoperative, and postoperative management. In addition, data on recipient selection, causes of death, and complications were not available from the transplantation centers. Recipient selection could certainly skew the data, but these limitations were beyond the control of our OPO. We are currently gathering data regarding recipient posttransplantation pulmonary function, incidence of obliterative bronchiolitis, and causes of death, but the data are only available for those recipients who have undergone transplantation within the last 6 month and are therefore not reported.

In the United States the most frequently reported indications for lung transplantation are emphysema or chronic obstructive pulmonary disease, cystic fibrosis, and pulmo- 


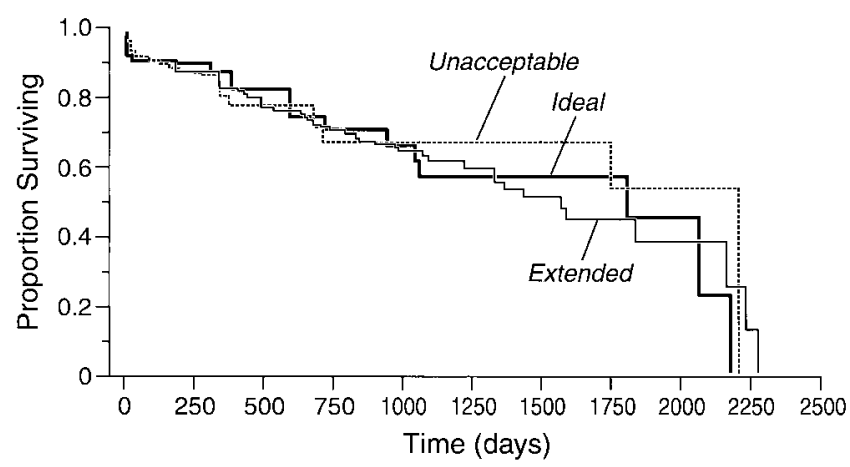

Figure 1. Kaplan-Meier survival curves comparing recipients of lungs from unacceptable, acceptable, and ideal donors. Note that there were no significant survival differences by donor group.

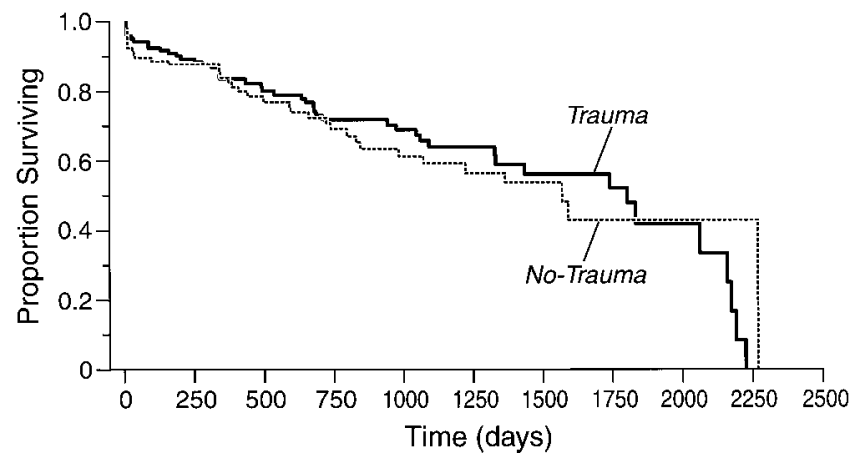

Figure 2. Kaplan-Meier survival curves comparing recipients of lungs from donors who died of trauma and those who died of nontraumatic causes. Note that there were no significant survival difference by donor group.

TABLE 6. Recipient survivals* with unacceptable, extended, and ideal donor lungs

\begin{tabular}{|c|c|c|c|c|}
\hline Type of transplant & $\begin{array}{l}\text { Recipients of lungs from } \\
\text { unacceptable donor group }\end{array}$ & $\begin{array}{l}\text { Recipients of lungs from } \\
\text { extended donor group }\end{array}$ & $\begin{array}{l}\text { Recipients of lungs from } \\
\text { ideal donor group }\end{array}$ & $P$ valuet \\
\hline All recipients & & & & .70 \\
\hline Median & 2202 & 1575 & 1810 & \\
\hline 95\% Confidence interval & $706-2202$ & 1224-2172 & 1046-2187 & \\
\hline Lung transplants & & & & .59 \\
\hline Median & 2202 & 1368 & 2073 & \\
\hline 95\% Confidence interval & $706-2202$ & 1093-1893 & $721-2073$ & \\
\hline Heart-lung transplants & & & & .58 \\
\hline Median & $\ddagger$ & 2172 & 1810 & \\
\hline 95\% Confidence interval & $\ddagger$ & $736-2281$ & $334-2187$ & \\
\hline
\end{tabular}

${ }^{*}$ Survival by Kaplan-Meier analysis.

†By log-rank test.

‡Unacceptable donor group was not considered for heart-lung transplants.

TABLE 7. Survivals* of recipients by donor cause of death

\begin{tabular}{llcc}
\hline & $\begin{array}{c}\text { Traumatic } \\
\text { cerebral deaths } \\
(\mathbf{n}=\mathbf{1 2 2})\end{array}$ & $\begin{array}{c}\text { Nontraumatic } \\
\text { cerebral deaths } \\
(\mathbf{n}=\mathbf{1 0 6})\end{array}$ & $\begin{array}{c}\boldsymbol{P} \\
\text { valuet }\end{array}$ \\
\hline Median & 1812 & 1575 & .86 \\
95\% Confidence interval & $1334-2172$ & $981-2281$ &
\end{tabular}

*Survival in days by Kaplan-Meier analysis.

†By log-rank test.

nary fibrosis. ${ }^{15}$ The shortage of donor organs has been the critical limiting factor in the treatment of end-stage lung disease. Although there has been an increase in the number of patients on the transplant waiting list, there has not been a corresponding increase in the number of available donor lungs. ${ }^{16-18}$ Furthermore, the AOPO documented an abysmally low rate of lung procurement from available donors, averaging only $13.4 \%$ among the 25 largest OPOs in 2000. Of more concern was the wide variability among OPOs with respect to lung procurement rates, suggesting that donor management may have dramatic effects on procurement success. Although the use of unsuitable organs would be catastrophic, many investigators are challenging the severe limitations imposed on lung transplant donor selection by what some consider arbitrary and rigid lung donation standards. ${ }^{6-8}$

Traditional lung transplant donor selection criteria for ideal candidates are listed in Table 1. Bhorade and colleagues ${ }^{6}$ demonstrated that extending their donor criteria to include what they called "marginal" donors did not adversely affect either short- or long-term recipient survival outcomes. They defined marginal donors as having any one of the following criteria: age older than 55 years, tobacco history of more than 20 pack-years, presence of infiltrate on chest radiograph, ventilator time longer than 5 days, or donor use of inhaled drugs (cocaine or marijuana). Interestingly, oxygenation was one criterion on which these investigators did not compromise. Each of the marginal donors maintained $\mathrm{PaO}_{2} / \mathrm{FIO}_{2}$ ratios greater than 350 at procurement. Bhorade and colleagues ${ }^{6}$ found no significant differ- 
TABLE 8. Pretransplantation diagnoses among lung transplant recipients

\begin{tabular}{|c|c|c|c|}
\hline Diagnosis & $\begin{array}{l}\text { Recipients of lungs from } \\
\text { unacceptable donor group }\end{array}$ & $\begin{array}{l}\text { Recipients of lungs from } \\
\text { ideal donor group }\end{array}$ & $\begin{array}{l}\text { Recipients of lungs from } \\
\text { acceptable donor group }\end{array}$ \\
\hline \multicolumn{4}{|c|}{$\begin{array}{l}\text { Chronic obstructive pulmonary disease and emphysema } \\
\text { (includes } \alpha_{1} \text {-antitrypsin deficiency) }\end{array}$} \\
\hline No. & 15 & 12 & 57 \\
\hline$\%$ & 44.2 & 27.9 & 37.8 \\
\hline \multicolumn{4}{|c|}{ Cystic fibrosis } \\
\hline No. & 7 & 7 & 20 \\
\hline$\%$ & 20.6 & 16.3 & 13.2 \\
\hline \multicolumn{4}{|c|}{ Pulmonary hypertension (includes Eisenmenger disease) } \\
\hline No. & 4 & 15 & 46 \\
\hline$\%$ & 11.8 & 34.9 & 30.4 \\
\hline \multicolumn{4}{|c|}{ Pulmonary fibrosis } \\
\hline No. & 8 & 8 & 25 \\
\hline$\%$ & 23.5 & 18.6 & 30.4 \\
\hline \multicolumn{4}{|c|}{ Retransplantation } \\
\hline No. & 0 & 1 & 3 \\
\hline$\%$ & 0 & 2.3 & 2.0 \\
\hline \multicolumn{4}{|c|}{ Total recipients } \\
\hline No. & 34 & 43 & 151 \\
\hline$\%$ & 100 & 100 & 100 \\
\hline
\end{tabular}

ence in 30-day and 1-year outcomes between the recipients of lungs from expanded donors (marginal lungs) and recipients of lungs from ideal donors. At 1 year after transplantation, the investigators noted a trend toward decreased pulmonary function in patients who received a single marginal donor lung, although this trend did not attain statistical significance. They therefore concluded that certain expanded criteria did not preclude successful transplantation, which would argue for a potential increase in the number of available donors. ${ }^{6}$

Sundaresan and associates ${ }^{7}$ also reviewed their experience with marginal donors. Their marginal group included 37 donors who failed to meet one traditional ideal donor criteria and 7 donors who failed to meet two criteria. Six donors in their marginal group had preoperative $\mathrm{PaO}_{2} / \mathrm{FIO}_{2}$ ratios less than 300, although their actual values were not defined. They made no aggressive attempts before procurement to improve the oxygenation of these donor lungs. They noted no difference in early oxygenation, length of ventilator support, and 30-day survivals when they compared recipients who received marginal lungs with those who received ideal lungs. Long-term follow-up was not available in their study, however, which leads to speculation about delayed pulmonary function and survival of these recipients.

Transplantation of lungs from donors involved in major trauma has traditionally been avoided because pulmonary dysfunction (often delayed in presentation) is a common sequela of major trauma. In addition to the initial pulmonary injury (contusion, laceration, and aspiration), circulating platelet and protein microaggregates are taken up by the
TABLE 9. Recipient survival relationship to donor group

\begin{tabular}{lccc}
\hline & Relative hazard & $\begin{array}{c}\text { 95\% Confidence } \\
\text { interval }\end{array}$ & $\boldsymbol{P}$ value \\
\hline Unacceptable donors & 0.64 & $0.29-1.41$ & .27 \\
Extended donors & 0.93 & $0.54-1.62$ & .80 \\
\hline
\end{tabular}

Cox proportional hazard regression analysis for recipient diagnosis (as listed in Table 8). Reference group was ideal donor group.

pulmonary microcirculation, which can lead to endothelial injury by neutrophil activation. ${ }^{19,20}$ Aggressive intravenous fluid administration during the initial resuscitation only compounds the alveolar injury and may cause pulmonary edema. Waller and coworkers ${ }^{21}$ demonstrated that donor death by trauma did not affect the following indices of graft function at 30 days: length of postoperative ventilation, $\mathrm{PaO}_{2} / \mathrm{FIO}_{2}$ ratios at 1 hour and 24 hours, incidence of diffuse alveolar damage in lung biopsy specimens at 7 days, and 30-day mortality. However, $30 \%$ of their early recipient mortality was directly related to the clinical condition of the donor at the time of transplantation. Specifically, death from gram-negative bacterial pneumonia, in which the causative organisms were present at donor bronchoalveolar lavage, could potentially have been prevented. They concluded that using donor lungs from patients who die of trauma does not in itself compromise early graft outcome, provided that appropriate management strategies are used, including antibiotic treatment on the basis of bronchoscopy cultures after transplantation. Our study corroborates this and demonstrates no difference in 30-day and 1-year survivals of 
lung grafts between those from donors who died of trauma and those who died of nontraumatic causes. In addition, we found that a greater number of our unacceptable donors were those who died of trauma, suggesting that the early lung dysfunction associated with trauma may be completely reversible.

Irrespective of the cause of death, most donors display some lung dysfunction before procurement. Kron and associates $^{8}$ recently hypothesized that mild pulmonary dysfunction was common among potential lung donors and should be treated after lung transplantation. They performed a prospective study that required personal evaluation by the transplant surgeons of all potential thoracic organ donors. All donors met certain criteria: $\mathrm{PaO}_{2} / \mathrm{FIO}_{2}$ ratio less than 350 but more than 300 , presence of infiltrate on radiograph, or purulent secretions at bronchoscopy. Kron and associates ${ }^{8}$ accepted lungs from donors who had been receiving mechanical ventilation for less than 48 hours whose secretions could be cleared with suction and whose Gram stain did not reveal fungus. Antibiotic regimens were tailored to the organisms found on Gram stain from bronchoscopy specimens and strictly adhered to after culture results. They confirmed that minor infiltrates from atelectasis and secretions could be treated after transplantation and that minor abnormalities in gas exchange could be corrected after transplantation by appropriate fluid and ventilator management. Long-term follow-up, however, was not available in their study.

Successful lung procurement can be optimized with aggressive donor management. The most common reasons for loss of donor lungs for transplantation are pulmonary edema, pulmonary sepsis, and contusions. ${ }^{22}$ Cummins and colleagues ${ }^{13}$ recently evaluated their donor management strategies. Their protocols focused on maximized ventilatory support, judicious fluid management, and maintenance of aggressive pulmonary toilet. Crystalloid rather than colloid fluids were administered, with targets of urinary output greater than $100 \mathrm{~mL} / \mathrm{h}$, systolic blood pressures greater than $100 \mathrm{~mm} \mathrm{Hg}$, and CVPs of 6 to $8 \mathrm{~mm} \mathrm{Hg}$. Vasopressors were used as needed to maintain organ perfusion. The goals of ventilator therapy were to maintain adequate oxygen saturation with minimal $\mathrm{FIO}_{2}$, to provide adequate ventilation to maintain normal acid-base balance, and to prevent inadequate ventilation and atelectasis. Chest physiotherapy was performed hourly, including sterile saline solution lavage and hyperventilation. Sputum Gram stains were obtained early, and antibiotic treatment was tailored to those results. A dramatic increase in lung procurement was demonstrated when these guidelines were followed. The retrieval rate of $33 \%$ reported by Cummins and colleagues ${ }^{13}$ was better than that of the largest centers reported by the AOPO. ${ }^{3}$

The 27 unacceptable donors would have been rejected by OPO protocol as lung donors at their initial evaluation because of their poor oxygenation. We do not know how many potential donors during the study period had a $\mathrm{PaO}_{2} /$ $\mathrm{FIO}_{2}$ ratio less than 150 that could not be optimized, and this presents a limitation of this study. However, we have shown that the aggressive use of CVP monitoring, diuresis, steroids, and vasopressors allowed each of our 27 donors in the unacceptable group to have their lungs successfully transplanted. No untoward effects could be attributed to this effort, and the number of recipients surviving was comparable with the survival of all other recipients, including those who received ideal organs. We also demonstrated that the recipient diagnosis and whether the recipient received an organ from this unacceptable group did not adversely affect the recipient outcome. At a time when donor organs are so precious, every effort must be made to optimize donor selection. Our study shows that special attention needs to be placed on donor management, especially for donors who die of trauma, because many of the initial insults are reversible with aggressive intervention. Early and long-term survivals of recipients of lungs from persons who died of trauma were not significantly different from those who died of nontraumatic causes. We do not know at present whether donor trauma will affect the late results of lung transplant recipients at 5 years after transplantation. We will be following up these patients carefully to try and answer this question.

We believe that one way to increase the number of acceptable lungs for transplantation is by the widespread use of aggressive management protocols by all OPOs. The wide variability seen among OPOs in terms of lung procurement rates may be lessened if standard management criteria are adopted and used. We also believe that all OPOs should define their pool of potential lung donors to exclude older and younger patients who would never be considered candidates for lung donation, thus ensuring a more accurate means of comparing OPO performance. It is our belief that if lung retrieval rates could be increased to a constant level nationally, consistent with the level in the most successful OPOs, there would be a large increase in the number of lungs available for transplantation.

\section{References}

1. Current estimates from the National Health Interview Survey 1993. Vital Health Stat 10. 1994;190:1-221.

2. 2000 Annual Report: US Scientific Registry of Transplant Recipients and The Organ Procurement and Transplantation Network. Transplant data 1990-1999. Washington, DC: US Department of Health and Human Services; 2000.

3. Association of Organ Procurement Organizations. OPO voluntary survey on local organ donor and transplantation activity for 12 month totals, annual report. McLean (VA): The Association; 2000.

4. Follette D, Rudich S, Bonacci C, Allen R, Hoso A, Albertson T. Importance of an aggressive multidisciplinary management approach to optimize lung donor procurement. Transplant Proc. 1999;31:16970 .

5. Armstrong GT. Utilization of donated lungs in Australia: 1989-1997. J Transpl Coord. 1999;9:181-5. 
6. Bhorade SM, Vigneswaran W, McCabe MA, Garrity ER. Liberalization of donor criteria may expand the donor pool without adverse consequence in lung transplantation. J Heart Lung Transplant. 2000; 19:1199-204.

7. Sundaresan S, Semenkovich J, Ochoa L, Richardson G, Trulock EP, Cooper JD, et al. Cardiac and pulmonary replacement: successful outcome of lung transplantation is not compromised by the use of marginal donor lungs. J Thorac Cardiovasc Surg. 1995;109:1075-79.

8. Kron IL, Tribble CG, Kern JA, Daniel TM, Rose CE, Truwit JD, et al Successful transplantation of marginally acceptable thoracic organs. Ann Surg. 1993;217:518-22.

9. Myoshi S, Schaefers HJ, Trulock EP, Yamazaki F, Schreinemakers H, Patterson GA, et al. Donor selection for single and double lung transplantation: chest size matching and other factors influencing posttransplantation vital capacity. Chest. 1990;98:308-13.

10. United Network for Organ Sharing. Guidelines for multiorgan donor management and procurement. UNOS Update. 1993:14-5.

11. Frost AE. Donor criteria and evaluation. Clin Chest Med. 1997;18: 231-7.

12. Wheeldon DR, Potter CD, Oduro A, Wallwork J, Large SR. Transforming the "unacceptable" donor: outcomes from the adoption of a standardized donor management technique. J Heart Lung Transplant. 1995; 14:734-42.

13. Cummings J, Houck J, Lichtenfeld D. Positive effect of aggressive resuscitative efforts on cadaver lung procurement. J Transpl Coord. 1995;5:103-6.

14. Sundaresan S, Trachiotis GD, Aoe M, Patterson GA, Cooper JD. Donor lung procurement: assessment and operative technique. Ann Thorac Surg. 1993;56:1409-13.

15. Keck BM, Bennett LE, Rosendale J, Daily OP, Novick RJ, Hosenpud JD. Worldwide thoracic organ transplantation: a report from the UNOS/ISHLT International Registry for Thoracic Organ Transplantation. Clin Transpl. 1999:35-49.

16. Trulock EP. Lung transplantation. Am J Respir Crit Care Med. 1997; 155:789-818.

17. Hosenpud JD, Bennett LE, Keck BM, FIol B, Novick JD. The Registry of the International Society for Heart and Lung Transplantation: fourteenth official report-1997. J Heart Lung Transplant. 1997;16: 691-712.

18. Keller CA. The donor lung: conservation of a precious resource. Thorax. 1998;53:506-13.

19. Shale DJ. The adult respiratory distress syndrome: 20 years on. Thorax. 1987;42:641-5.

20. Rivkind AI, Siegel JH, Guadalupi P, Littleton M. Sequential patterns of eicosanoid, platelet, and neutrophil interactions in the evolution of the fulminant post-traumatic adult respiratory distress syndrome. Ann Surg. 1989;210:355-72.

21. Waller DA, Thompson AM, Wrightson WN, Gould FK, Corris PA, Hilton CJ, et al. Does the mode of donor death influence the early outcome of lung transplantation? A review of lung transplantation from donors involved in major trauma. J Heart Lung Transplant. 1995; $14: 318-21$.

22. Malen JF, Ochoa LL, Sander MC, Straatman D. Lung transplantation. Crit Care Nurs Clin North Am. 1992;4:111-30.

23. United Network for Organ Sharing. The OPTN data as of May 12, 2001: annual report of UNOS data. Richmond (VA): UNOS. 2000.

\section{Discussion}

Dr Robert C. Robbins (Stanford, Calif). This study was designed to test the hypothesis that donors with unacceptable $\mathrm{PaO}_{2} / \mathrm{FIO}_{2}$ ratios of less than 150 could become acceptable lung donors with aggressive OPO management and that the 30-day and 1-year outcomes would be no different from the results achieved with traditional ideal donors. Straznicka and coworkers also investigated the results of pulmonary transplants from donors who had sustained traumatic injuries and compared them with the results of transplants from donors without a history of traumatic injury.

This article addresses an important area of investigation, because there is a severe imbalance in the supply of donor lungs for the demand of patients awaiting transplantation. This imbalance in pulmonary donor organs is the most severe of all transplanted organs, with the best procurement average by any OPO in the United States at approximately $30 \%$. Therefore any attempt to increase the number of donor lungs is a laudable objective.

Straznicka and coworkers report that of the 194 donors from their OPO who donated lungs, resulting in 224 transplants, 27 donors were deemed unacceptable for lung donation because of oxygenation values less than the standard threshold $\mathrm{PaO}_{2} / \mathrm{FIO}_{2}$ ratio of 150 . They demonstrated that 1 -month and 1-year survivals were not statistically significantly different from results obtained with initially acceptable lung donors. Paradoxically, in fact, the survivals of patients who received lungs from unacceptable lung donors were better than those who received lungs from an ideal subset of the acceptable donors. There is no question that donors with initially poor oxygenation can be effectively managed to provide excellent donor lungs. Furthermore, Straznicka and coworkers provide evidence that these lungs will support recipients for as long as 1 year after transplantation.

I have a few observations about the study. It is limited by the small number of patients and its retrospective nature. The statistical comparison of survivals at 1 month and 1 year by the Student $t$ test is not valid, and an actuarial analysis such as Kaplan-Meier method with log-rank testing for significance is needed. The comparison of results between the groups is problematic, because the recipient diagnoses were not accounted for in this analysis. It is possible that the initially unacceptable lungs were transplanted into the best recipients and that the ideal donor lungs were placed into recipients at higher risk. We know that this situation partially exists in this study, because the survival in the ideal group was the lowest in all the groups and this was the only group that supplied lungs to heart-lung recipients. Certainly many recipient factors that affect outcome are independent of the quality of lungs during the procurement process. The $\mathrm{PaO}_{2} / \mathrm{FIO}_{2}$ ratio was the only discriminator used to include donors in the unacceptable group. The optimization maneuvers used were generally procedures that are standard with good donor management, such as diuresis, bronchoscopy, and monitoring of chest radiographs. The use of steroids is the only factor that was outside the standard of care, and this is now part of the routine management of all prospective lung donors in this OPO. Maybe these groups were really quite similar, and the initial $\mathrm{PaO}_{2} / \mathrm{FIO}_{2}$ ratio is a poor predictor of outcome when it is the only factor considered. The 1-month and 1-year results were excellent in the unacceptable cohort, but we have no information about either short-term or long-term recipient pulmonary function. Specifically, the incidence of obliterative bronchiolitis is unknown, and this may prove to be an important factor, especially in the traumatically injured donor group. Finally, we must remember that at the time of procurement the donor lungs in the unacceptable group were not only acceptable but trending toward ideal, with excellent oxygenation and clear chest radiographs.

Dr Straznicka, I have a few questions. What was the denominator for the 27 patients in the unacceptable group? Specifically, how many of the 1000 potential lung donors in your OPO during the study period had $\mathrm{PaO}_{2} / \mathrm{FIO}_{2}$ ratios less than 150 that could not be optimized?

Dr Straznicka. We do not have those data, but it is something that we will look into in the future. 
Dr Robbins. I suggest that if we were to analyze the data a bit more carefully, we could learn a lot of valuable information that could predict which potential donors could have optimization performed, which could allow us to further expand the donor pool for lungs into more marginal donors.

Was the $\mathrm{PaO}_{2} / \mathrm{FIO}_{2}$ ratio that you reported an initial value reported to the OPO, or was it the lowest or some average of the values?

Dr Straznicka. It was the initial $\mathrm{PaO}_{2} / \mathrm{FIO}_{2}$ ratio. The initial $\mathrm{PaO}_{2} / \mathrm{FIO}_{2}$ ratio was the one shortly after brain death declaration, when the decision was made whether the patient could be a potential lung donor.

Dr Robbins. Do you have any information about whether in the acceptable donor groups this $\mathrm{PaO}_{2} / \mathrm{FIO}_{2}$ ratio was less than 150 at any time during the hospitalization? Is it possible that some of the acceptable donors had a $\mathrm{PaO}_{2} / \mathrm{FIO}_{2}$ ratio less than 150 before the OPO ever began management? Do you know whether that is true, or were they always good gas values?

Dr Straznicka. All the blood gas values in the acceptable donor group showed a $\mathrm{PaO}_{2} / \mathrm{FIO}_{2}$ ratio greater than 150 during the entire period of management.

Dr Robbins. How many patients in the acceptable group had one or more unacceptable criteria, such as age older than 55 years, smoking history, abnormal sputum, or chest trauma? Do you have any idea about information about the patients in the acceptable group? Were there some unacceptable things about them?

Dr Straznicka. The acceptable group did in fact have one or more criteria that were not part of the ideal criteria, and for that reason they were not among the 38 ideal donors, but I do not have the exact breakdown of which of those criteria each donor had.

Dr Robbins. What were the causes of death among the recipients? There were only a few deaths, but were any of the deaths related to the poor quality of donor lungs? Do you have any idea about that?

Dr Straznicka. We do not have information from the transplantation centers on the cause of death of the recipient and whether it was related to donor factors.

Dr Robbins. In summary, there is an important message in this article, and that is do not turn down lungs for transplantation just because of one blood gas value. Approximately $30 \%$ of young donors nationwide have their hearts refused for transplantation on the basis of poor function from one echocardiogram. There are certainly many factors that are released during the process of brain death that serve as negative inotropic agents and contribute to neurogenic pulmonary edema, and as we have heard today many of these organs can be salvaged.

A recent conference sponsored by the American Society of Transplant Surgeons and organized by Bruce Rosengard of the University of Pennsylvania and attended by a couple of the coauthors of this article explored the various methods that one might use to increase donor use nationwide. There are several prospec- tive protocols that are currently being written and will be implemented to try to achieve this goal.

The highest rates of OPO procurement of lungs are found in areas where there is an active lung transplant program and good communication between the center and the OPO. It certainly is true that the OPO personnel need to believe that aggressive management of these donor lungs will likely produce lungs that we will be able to transplant, but they also must have the confidence that the surgeons will use these lungs and be receptive to this aggressive management.

Dr Michael Mulligan (Seattle, Wash). Articles in the literature that look at the use of marginal lung donors include the series from Australia, the Chicago experience out of Loyola, the University of Virginia experience from Irving Kron's group, the article from Toronto recently discussed at the American Association for Thoracic Surgery meeting, and a couple of different reports that have come out of St Louis. No group used an initial $\mathrm{PO}_{2}$ as a criterion for defining a marginal donor. Everybody used a final $\mathrm{Po}_{2}$, and many of those donors who were considered ideal had a suboptimal $\mathrm{PO}_{2}$ at some point. Given that, unless the donors you defined as marginal had purulent sputum on bronchoscopy, significant smoking history, or age older than 55 years, all of those donors would have been considered ideal according to the authors of those other studies. I therefore do not find it entirely surprising that the results you presented are good.

It may also be that we need to look at these recipients a bit further out. As you know with significant acute graft dysfunction, major histocompatibility complex class II expression is much elevated and they are not only at risk for early morbidity but they may be at risk for increased difficulty with obliterative bronchiolitis long term.

How many of the donors defined as unacceptable at first were older than 55 years, had significant smoking, or had purulent bronchoscopy, because all their final chest radiographs were clear?

Dr Straznicka. We have not looked at the data in terms of which of the extended criteria you mentioned were present in the acceptable group of donors. That is something that we will look at in the future.

Dr Mulligan. I also wish to underscore the point of Dr Robbins about the importance of recipient diagnosis. Groups that have used marginal donors, truly marginal donors, for patients with persistent pulmonary hypertension, cystic fibrosis, or idiopathic pulmonary fibrosis have gotten into a lot of trouble. Conversely, even lungs with a final $\mathrm{PO}_{2}$ less than $300 \mathrm{~mm} \mathrm{Hg}$, purulent secretions that are unilateral, or a unilateral dense infiltrate can be used as a doublelung block for a recipient with chronic obstructive pulmonary disease with excellent results, specifically 5-year survivals that appear to be in excess of $60 \%$. Ultimately, we do want to use these marginal lungs, but we may want to be careful about recipient selection for them. 